\title{
44361 - PROBING THE IDENTITY OF GABAA RECEPTORS THAT UNDERLIE AMNESTIC PROPERTIES OF ANESTHETICS
}

\author{
Angelina Guzzo, Sunnybrook Health Sciences Centre, Toronto, ON, Canada; \\ William Ju \\ Mary Chiu \\ P Taylor, Hospital for Sick Children; \\ M Moran, Hospital for Sick Children and University of Toronto; \\ BA Orser, University of Toronto;
}

INTRODUCTION: The desirable components of the anesthetic state include amnesia, hypnosis, immobility and analgesia. Different anatomical regions and receptors are thought to mediate these distinct behavioral end-points. In order to design specific drugs, it is essential to know the protein structure of anesthetic targets. Our aim is to identify specific receptors that underlie the amnestic properties of anesthetics. GABAA receptors which mediate most inhibitory neurotransmission are targets for injectable and inhaled anesthetics. GABAA receptors are pentameric complexes that are composed of multiple subunits (\&\#945;1-6, \&\#946;1-3, \&\#947;14, \&\#948;, \&\#961;1-3, \&\#963;, \&\#960;) which determine physiologic properties and sensitivities to anesthetics $(1,2)$. In particular, GABAA receptors that contain the $\& \# 945 ; 5$ subunit play a critical role in learning and memory $(3,4)$. Genetic deletion of the \&\#945;5 subunit conferred resistance to the amnestic properties of the anesthetic etomidate in a mouse model, whereas sedation and hypnosis remained intact (5). The goal of this study was to identify the subunits that combine with the \&\#945;5 subunit to form functional GABAA receptors in hippocampal neurons.

METHODS: Studies were approved by the local Animal Care Committee. C57/BL6 mouse hippocampi were isolated and fractionated to enrich for synaptosomal and nonsynaptosomal protein complexes. Immunoprecipitation was performed using a polyclonal antibody raised against the major intracytoplasmic loop of the GABAA receptor \&\#945;5 subunit (Sigma, Saint Louis, MO). The complexes were fractionated by size using SDS-gel electrophoresis and purified. After trypsin digestion, proteins were identified using mass spectrometry.

RESULTS: Mass spectrometry showed that \&\#945;5 subunit associates with not one \&\#945; or \&\#946; isoform, but rather with a diverse number of subunits including \&\#945;1, \&\#945;2, \&\#946;1, \&\#946;3 and \&\#947;2.

DISCUSSION: The results provide the first direct evidence that GABAA receptors that contain the \&\#945;5 subunit represent a heterogenous population which incorporate several \&\#945; and \&\#946; isoforms. Since current models suggest that individual GABAA receptor isoforms mediate the specificity of GABA's physiological effects, the results predict that \&\#945;5 GABAA containing receptors will exhibit diversity in their trafficking (6), pharmacology and physiological properties $(1,2)$. The results change our basic understanding of these receptors and will modify our approach to future drug design. 


\section{REFERENCES:}

(1) Cell Tissue Res 2006 326:505-516

(2) Curr Opinion Pharm 2006 6:18-23

(3) J Neurosci 2002 22: 5572-80

(4) Proc Natl Acad Sci.USA 2004 101:3662-7

(5) J Neurosci 2006 26:3713-3720

(6) Pharmacol Ther 2003 98:299-323 